Notfall Rettungsmed 2017 $\cdot 20: 639-640$ https://doi.org/10.1007/s10049-017-0388-1 Online publiziert: 15. November 2017

(c) Springer Medizin Verlag GmbH 2017

\section{T. Nicolai' ${ }^{1}$ H. Dormann ${ }^{2}$}

'Dr. von Haunersches Kinderspital Kinderklinik und Kinderpoliklinik, Ludwig Maximilian Universität München, München, Deutschland

${ }^{2}$ Zentrale Notaufnahme, Klinikum Fürth, Fürth, Deutschland

CrossMark

\title{
Nichtinvasive Beatmung (NIV) bei akutem respiratorischem Versagen
}

Das akute Atemversagen stellt eine typische Ursache für die Inanspruchnahme des Notarztes oder einer Notaufnahme dar. Nicht selten bestehen gleichzeitig andere pathologische Veränderungen, die von der richtigen Auswahl der respiratorischen Unterstützungsmethode günstig beeinflusst werden können.

Hier hat die nichtinvasive Beatmung (NIV) einen wichtigen Platz in der Notfallmedizin gefunden. Obwohl seit Ende der 1980er Jahre die Vorteile einer NIV bei Notfallpatienten mit akuter respiratorischer Insuffizienz bekannt sind und seitdem zahlreiche Studien eindeutige Empfehlungen zur Anwendung geben, wird diese Methode im Rettungsdienst, der Notaufnahme und bei Kindern nicht konsequent umgesetzt. Ziel dieser Maßnahme kann es neben der Sicherstellung der Ventilation und der Verbesserung des Gasaustausches sein, Zeit zu gewinnen: um entweder die der Ateminsuffizienz zugrunde liegenden Prozesse zu identifizieren und therapeutisch so zu beeinflussen, dass eine invasivere respiratorische Unterstützung gar nicht mehr notwendig wird, oder bei eindeutiger Indikation eine invasive Beatmung einzuleiten.

Bei Kindern wie Erwachsenen besteht in der Atemnotsituation eine eingeschränkte Kooperationsfähigkeit. Auch stellen stark variierende Körperproportionen bezüglich der Auswahl optimaler Devices eine besondere Herausforderung dar. Entscheidend für den Erfolg einer NIV ist aber, dass vom Notarzteinsatzort, über die Notaufnahme bis hin zur Intensivstation eine lückenlose NIV-Versorgungskette gelebt wird. Die
Sicherstellung der NIV bei akuter respiratorischer Insuffizienz bei Patienten mit pulmonalvenöser Stauung, bei exazerbierter COPD („chronic obstructive pulmonary disease"), bei immunsupprimierten Patienten oder auch in der neonatologischen Notfall- und Intensivmedizin zeigen gegenüber der invasiven Beatmung deutliche Überlebensvorteile.

\section{》) Entscheidend für den Erfolg einer NIV ist eine lückenlose NIV- Versorgungskette}

Die beste Versorgung wird also nicht automatisch mit der Anwendung invasiverer oder technisch komplexerer Therapieverfahren sichergestellt. Fuchs et al. zeigen in ihrem Artikel „Nichtinvasive Beatmung bei Kindern mit akuter respiratorischen Insuffizienz" das breite Einsatzgebiet für die nichtinvasive $\mathrm{Be}$ atmung bei Kindern auf. Kinder mit ihrer eingeschränkten Kooperationsfähigkeit und der außerordentlichen Variabilität von Größe und Form des Gesichts bieten besondere Probleme bei der Auswahl und Anpassung des Beatmungsinterfaces. Neue technische Entwicklungen, bessere Materialien und mehr Erfahrung haben hier jedoch große Fortschritte ermöglicht.

Der Zustand von Kindern kann sich jedoch insbesondere in der Initialphase eines ARDS („acute respiratory distress syndrome“) oder einer Sepsis schlagartig so verschlechtern, dass notfallmäßig der Übergang zu einer invasiven Beatmung erforderlich wird. Wird hierbei zu lan- 
ge gewartet, kann die Etablierung eines invasiven Atemwegs zu schwerster Instabilität bis zum Atem-Kreislauf-Stillstand der Patienten führen. Eine NIVBeatmung beim kritisch kranken Kind sollte also nur an entsprechend erfahrenen und ausgestatteten Zentren erfolgen.

Den Anspruch, NIV in der Notfallund Rettungsmedizin beim Erwachsenen durchführen zu können, sollte heute jeder Rettungsdienst und jede Notaufnahme haben. Die Evidenz ist eindeutig, die Machbarkeit aber leider noch nicht. In den Artikeln zur „NIV in der Notaufnahme" von Dormann et al. und dem Artikel „NIV im Notarzt- und Rettungsdienst" von Sellmann et al. werden ausgehend vom Status quo Indikationen und Kontraindikationen benannt und neben technischen Möglichkeiten auch Teamaspekte benannt, die zur sicheren Anwendung der NIV beitragen.

Dieses Sonderheft stellt in einzigartiger Weise eine zusammenfassende Übersicht der NIV vom Kind bis zum Erwachsenen und vom Einsatzort des Rettungsdienstes über die Notaufnahmen bis zu Kinderintensivstation dar.

Wir hoffen, mit diesem Themenheft einen Beitrag zur weiteren Etablierung dieser segensreichen Behandlungsmethode leisten zu können

\section{Muerer}

T. Nicolai

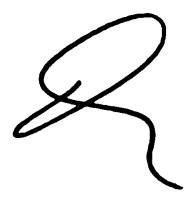

H. Dormann

\section{Korrespondenzadresse \\ Neurologie in der Notaufnahme}

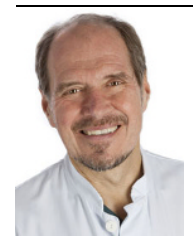

Prof. Dr. T. Nicolai

Dr. von Haunersches

Kinderspital Kinderklinik und

Kinderpoliklinik, Ludwig

Maximilian Universität

München

Lindwurmstr. 4, 80337 Mün-

chen, Deutschland

Thomas.Nicolai@

med.uni-muenchen.de

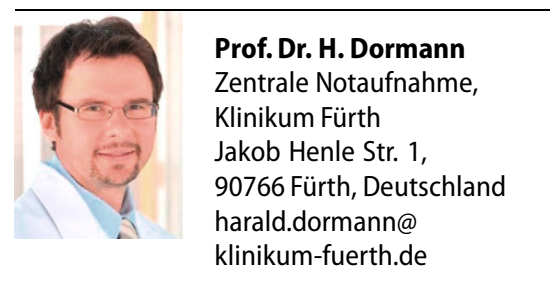

Interessenkonflikt. T. Nicolai und H. Dormann geben an, dass kein Interessenkonflikt besteht.

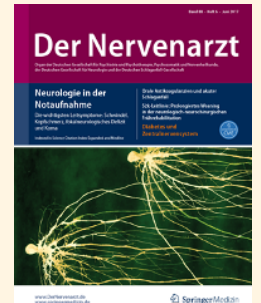

In großen interdisziplinären Notaufnahmen weisen etwa $15 \%$ aller Notfallpatienten neurologische Beschwerden auf mit steigender Tendenz. Diese

Entwicklung unterstreicht die Wichtigkeit von neurologischen Grundkenntnissen bei notärztlich tätigen Nicht-Neurologen und macht die fachärztliche neurologische Expertise in Notaufnahmen unabdingbar. Nicht selten lassen sich nicht nur neurologische sondern auch internistische Diagnosen als ursächlich für ein neurologisches Leitsymptom identifizieren.

In Der Nervenarzt 06/2017 liegt in allen Übersichtsarbeiten der Fokus auf einer symptomorientierten und ressourcenoptimierenden Herangehensweise mit praktischen Empfehlungen für Ärzte verschiedener Fachrichtungen

- Schwindel in der Notaufnahme

- Kopfschmerz in der Notaufnahme

- Koma in der Notaufnahme

- Akutes fokalneurologisches Defizit in der Notaufnahme

\section{Suchen Sie noch mehr zum Thema?} Mit e.Med - den maßgeschneiderten Fortbildungsabos von Springer Medizin - haben Sie Zugriff auf alle Inhalte von SpringerMedizin.de. Sie können schnell und komfortabel in den für Sie relevanten Zeitschriften recherchieren und auf alle Inhalte im Volltext zugreifen.

Weitere Infos zu e.Med finden Sie auf springermedizin.de unter "Abos" 